\title{
BMJ Open Internet-based cognitive-behavioural therapy for prevention of depression during pregnancy and in the post partum (iPDP): a protocol for a large- scale randomised controlled trial
}

\author{
Daisuke Nishi (D) , ${ }^{1}$ Kotaro Imamura, ${ }^{1}$ Kazuhiro Watanabe, ${ }^{1}$ Erika Obikane, ${ }^{1}$ \\ Natsu Sasaki, ${ }^{1}$ Naonori Yasuma (D) , ${ }^{1}$ Yuki Sekiya, ${ }^{1}$ Yutaka Matsuyama, ${ }^{2}$ \\ Norito Kawakami (D) ${ }^{1}$
}

To cite: Nishi D, Imamura K, Watanabe $\mathrm{K}$, et al. Internetbased cognitive-behavioural therapy for prevention of depression during pregnancy and in the post partum (iPDP): a protocol for a large-scale randomised controlled trial. BMJ Open 2020;10:e036482. doi:10.1136/ bmjopen-2019-036482

- Prepublication history for this paper is available online. To view these files, please visit the journal online (http://dx.doi org/10.1136/bmjopen-2019036482).

Received 18 December 2019 Revised 16 March 2020 Accepted 03 April 2020

Check for updates

(c) Author(s) (or their employer(s)) 2020. Re-use permitted under CC BY-NC. No commercial re-use. See rights and permissions. Published by BMJ.

${ }^{1}$ Department of Mental Health, Graduate School of Medicine, The University of Tokyo, Tokyo, Japan

${ }^{2}$ Department of Biostatistics, Graduate School of Medicine, The University of Tokyo, Tokyo, Japan

Correspondence to Dr Daisuke Nishi; d-nishi@m.u-tokyo.ac.jp

\section{ABSTRACT}

Introduction The objective of this randomised controlled trial (RCT) is to examine the effects of smartphone-based cognitive-behavioural therapy (CBT) in preventing the onset of major depressive episodes (MDE) among pregnant women.

Methods and analysis The target study population will be pregnant women of 16-20 weeks gestation who are currently users of 'Luna Luna Baby', the most widely used app for pregnant women in Japan. Those who meet the eligibility criteria will be randomly allocated to the 6-module internet CBT programme that was newly developed for pregnant women $(n=2500)$, or to a treatment-as-usual control group $(n=2500)$. Participants in the intervention groups will be required to complete the programme by 32 weeks gestation. The primary outcomes are the number of new onsets of MDE, measured by using WHO Composite International Diagnostic Interview 3.0 at 32 weeks gestation and 3 months post partum. Survival analysis will be conducted to test for the effectiveness of the intervention on the time to the onset of MDE.

Ethics and dissemination The study plan has been approved by the Research Ethics Review Board of the Graduate School of Medicine/Faculty of Medicine, the University of Tokyo (2019150NI). If the intervention programmes are found to produce a significant positive effect in this RCT, these programmes can be made available for all users of the app in the future. Trial registration number UMIN000038190; Pre-results.

\section{INTRODUCTION}

Prevention of perinatal depression is a critical public health issue. ${ }^{1}$ The prevalence of antenatal depression was reported to be $7.4 \%$ in the first trimester, $12.8 \%$ in the second trimester and $12.0 \%$ in the third trimester ${ }^{2}$; and the prevalence of postpartum depression was estimated to be $17.7 \%$, though there is significant heterogeneity across nations. ${ }^{3}$ Antenatal depression is associated with failing to seek prenatal care, an inadequate

\section{Strengths and limitations of this study}

- The large sample size of this randomised controlled trial (RCT) can lead to a definitive result of the effectiveness of the fully automated smartphone-based cognitive-behavioural therapy (CBT) programme in preventing both antenatal and postpartum depression.

- The newly developed programme was tailored for pregnant women by extracting essential topics for them based on data from consultations on pregnant women's concerns.

- This study will also evaluate the implementation outcomes of internet CBT (iCBT) programme, which will contribute to the dissemination and implementation of iCBT.

- This RCT will also clarify whether iCBT during pregnancy can prevent maternity blues.

- A limitation of this study is that all of the outcomes will be measured by self-report.

diet and/or use of tobacco, alcohol, or other harmful substances, self-harm or attempted suicide, and postpartum depression, while postpartum depression is associated with negative outcomes such as physical and psychological abuse to their children, and infanticide. ${ }^{4}$ Furthermore, perinatal depression can also affect development from fetus to adolescent and paternal depression..$^{5-10}$ Therefore, preventing perinatal depression in the antenatal period is crucially important.

Given the large number of pregnant women who have risk factors such as maternal anxiety, life stress, lack of social support, unintended pregnancy, domestic violence, lower income and education, and poor relationships, making it difficult to identify and screen all high-risk pregnant women, ${ }^{11}$ 
universal prevention should be more valued. According to a systematic review and meta-analysis, psychological interventions have been recommended as the most effective approach to prevent antenatal and postpartum depression. $^{12}$ Cognitive-behavioural therapy (CBT) has been broadly researched and reported to be highly effective among psychological interventions. ${ }^{13}{ }^{14}$ Previous meta-analyses showed that the effect size of psychological intervention as universal prevention, which refers to approaches designed for the whole population regardless of individual risk factors, for postpartum depression was reported to be $0.19^{15}$ and 0.37 . $^{13}$ The timing of interventions of randomised controlled trials (RCTs) included in these meta-analyses was both during pregnancy and post partum.

With respect to universal prevention, fully automated internet-based CBT (iCBT) is preferable to face-to-face or guided iCBT in terms of accessibility, anonymity and cost effectiveness. ${ }^{16}$ Although a systematic review showed a positive effect of iCBT for the improvement of perinatal depressive symptoms, ${ }^{17}$ to our knowledge, there have been only two RCTs for universal prevention using automated iCBT during pregnancy. ${ }^{18} 19$ These two studies did not assess the major depressive episode (MDE); instead, they used depressive symptoms as outcomes. In addition, a dropout from iCBT in these studies was relatively high (66\% and $44 \%$, respectively). A large number of sessions and/or the length per session may be impractical for a substantial number of pregnant women to complete, which may cause a high dropout rate. Thus, it would be necessary to develop a programme that is more acceptable and feasible for pregnant women. In this regard, six sessions of 5-10 min iCBT programmes were shown to be effective for preventing depressive symptoms for workers. $^{20}$

Moreover, to our knowledge, no previous RCTs have examined the effect of iCBT on maternity blues. Maternity blues were characterised by psychological distress with a peak at 3-5 days after childbirth, though diagnostic criteria have not been well established. Maternity blues are highly prevalent and have been shown to be a risk factor for postpartum depression ${ }^{21}$; thus, it will be relevant to develop the intervention to prevent not only perinatal depression but also maternity blues.

The primary objective of this RCT is to examine the effectiveness of the newly developed six sessions of 5-10 min, smartphone-based, automated iCBT programmes in preventing the onset of MDE at the third trimester and 3 months post partum among pregnant women currently in the second trimester. The secondary objectives are to examine the effectiveness of iCBT for preventing maternity blues. The programme would be practical and could be used by many pregnant women. In addition, intervention during early pregnancy will enable the prevention of not only postpartum depression but also antenatal depression.

\section{METHODS AND ANALYSIS}

\section{Trial design}

The study will be a two-arm, parallel-group, treatmentas-usual (TAU) controlled, randomised trial. The allocation ratio of the intervention groups to the control group is 1:1. Random assignments are stratified by Kessler's Psychological Distress Scale (K6) scores (groups of four points or less and groups of five points or more) in the baseline survey. K6 is a self-report questionnaire, which assesses psychological distress during the past 30 days. Users of the app ('Luna Luna Baby' run by MTI) will be recruited. The app provides the users for the growth of the fetus and the mental and physical condition of the pregnant women according to the number of gestation weeks. Users register the date of the last menstruation in the app; thus, we can find out the number of weeks of pregnancy.

Follow-up assessments will be conducted at 32 weeks gestation and 3 months post partum. This protocol is written in accordance with the Standard Protocol Items: Recommendations for Interventional Trials guideline checklist. ${ }^{22}$ The trial start date was 27 November 2019, and the last follow-up date will be 31 March 2021.

\section{Participants}

Pregnant women who have user identifications for the latest version of the app and meet the following criteria will be invited to participate in this RCT. Both primipara and multiparous women will be included.

\section{Eligibility criteria}

1. Being over 20 years old.

2. Being 16-20 weeks gestation.

3. Not diagnosed with an MDE in the past month by the web-based self-administered version of WHO Composite International Diagnostic Interview 3.0 (WHO-CIDI $3.0){ }^{23}$

4. Not diagnosed with lifetime bipolar disorder (WHOCIDI 3.0).

\section{Recruitment}

Figure 1 shows the study flow of this trial. MTI sends an invitation message to potentially eligible pregnant women, which will include an explanation of the study and information on the eligibility criteria. After reading the explanation of the study, potential participants will be invited to give their consent on the app to participate in the study and to complete and return the baseline survey. MTI will also send messages to study participants to join in follow-up assessments.

Five thousand pregnant women will be randomised to either the intervention group $(n=2500)$, or the control group $(\mathrm{n}=2500)$. Participants in the intervention groups will be required to complete the intervention programme up to 32 weeks gestation. Participants will be asked not to share this information through any social network. The participants will be reminded by a popup message to complete the programme if they have not already done 


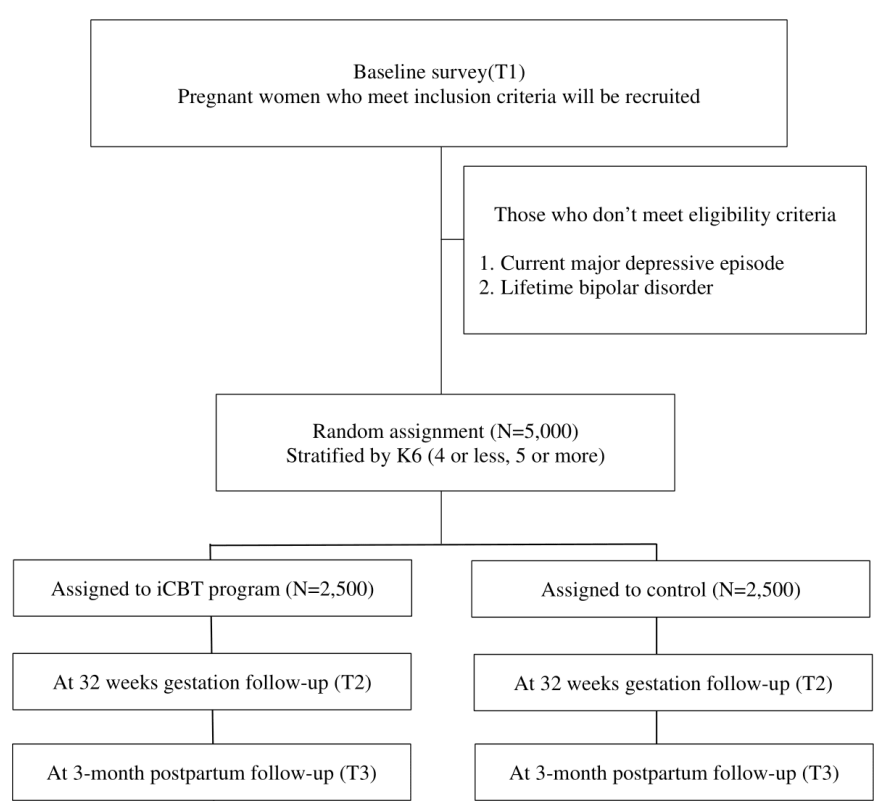

Figure 1 Flow diagram of study participants.

so. Intervention programmes will be closed at 32 weeks gestation.

Participants in the control group will not receive any intervention programmes during the intervention and follow-up period. General information about mental health during pregnancy will be provided to participants in both the intervention group and the control group as a TAU.

\section{Interventions}

A smartphone-based six-module CBT programme designed for pregnant women was newly developed. Specifically, the first author (DN) developed the iCBT programme with the collaboration of coauthors (KI, EO, NS and YS). The programme was tailored for pregnant women by extracting essential topics that pregnant women are concerned about. The details were shown in the Patient and public involvement section. This programme will be provided via the Luna Luna Baby app, so users of the app do not have to download another app. Some components of the modules were derived from a previous iCBT programme that successfully prevented the onset of MDE among office workers. ${ }^{20}$ Other components such as behavioural activation based on values, selfcompassion and mindfulness have been incorporated, which could be regarded as the third wave CBT. The six modules are presented in a fixed order, with one module accessible per week, from module 1 to module 6 (table 1 ). It will take about $5 \mathrm{~min}$ to complete each module. The programme includes psychoeducation (module 1), case formulation based on a cognitive-behavioural (CB) model (module 2), behavioural activation (module 3), self-compassion (module 4), mindfulness (module 5) and problem solving (module 6). Details of each of the components are as follows.
Table 1 Contents of internet cognitive-behavioural therapy programme

\begin{tabular}{|c|c|}
\hline Module no & Techniques for stress management \\
\hline Module 1 & Psychoeducation \\
\hline Module 2 & $\begin{array}{l}\text { Case formulation based on cognitive- } \\
\text { behavioural model }\end{array}$ \\
\hline Module 3 & Behavioural activation \\
\hline Module 4 & Self-compassion \\
\hline Module 5 & Mindfulness \\
\hline Module 6 & Problem solving \\
\hline
\end{tabular}

Psychoeducation (module 1)

In this module, participants learn about the roles of what are generally called 'negative' emotions such as anxiety, depressive mood and anger. Each emotion is necessary for us; for example, anxiety is a sign that warns us of some risk in the future and promotes us to prepare for the future. This module was designed to help participants face and deal with their own emotions in subsequent modules. As an example of anxious situations, a scene that a partner of a pregnant woman is busy working and is not at home is used. As an example of sad situations, a scene when a pregnant woman suffers from morning sickness but the boss does not understand is used.

\section{Case formulation based on CB model (module 2)}

In this module, participants learn about a CB model, especially the five-part model (situation, thoughts, emotions, behaviour and physical sensations) and a case formulation based on this model. ${ }^{24}$ Case formulation is a method used to understand the problems of patients or clients. Case formulation is helpful for participants to choose an appropriate approach to change the vicious circles of these five areas. A scene when a pregnant woman suffers from morning sickness but the boss does not understand is used as a case.

\section{Behavioural activation (module 3)}

Behavioural activation is a process to increase pleasurable and rewarding activities using behavioural strategies such as activity scheduling. This module provides a behavioural activation technique for enhancing participants' liveliness. In this module, participants learn about the theory of behavioural activation and how to plan an activity schedule to increase pleasant activities. Participants are also encouraged to identify their values based on brief behavioural activation therapy for depression, ${ }^{25}$ and acceptance and commitment therapy. ${ }^{26}$ A scene when a pregnant woman would not like to go out because she has gained weight and is not motivated is used as a case.

\section{Self-compassion (module 4)}

Self-compassion indicates a positive and caring attitude of a person towards herself in the face of stressful events. ${ }^{27}$ As a result of this attitude, highly self-compassionate individuals are expected to experience higher individual 
well-being. ${ }^{28}$ Three inter-related determine the selfcompassionate reactions to negative events and experiences: self-kindness, sense of common humanity and mindfulness. In this module, participants learn the concept of self-compassion and how to express compassion towards themselves. A scene when a pregnant woman suffers from morning sickness and is blaming herself for not being able to work as usual is used as a case.

\section{Mindfulness (module 5)}

Mindfulness is defined as "paying attention in a particular way: on purpose, in the present moment, and nonjudgmentally'. ${ }^{29}$ Mindful persons are likely to be aware of the physical sensation, thoughts and emotions at that moment, which enables them to stop their usual reactions to a stressful event so that symptoms and problematic behaviours are likely to disappear. In this module, participants learn about the concept of mindfulness and how to practice it through listening to voice guidance. A scene when a pregnant woman feels anxiety due to tension and pain in the lower abdomen in spite of obstetrically normal is used as a case.
Problem solving (module 6)

Problem-solving technique is a $\mathrm{CB}$ intervention that focuses on training adaptive problem-solving attitudes and skills. ${ }^{30}$ A rational problem-solving style contains the systematic application of four problem-solving skills: (1) problem definition and formulation, (2) generation of alternative solutions, (3) decision-making and (4) solution implementation and verification. In this module, participants learn about problem-solving skills to sort out the problem and make a list of solutions, and assertiveness to communicate with their partners confidently. A scene when a pregnant woman wants her partner to do more in housework and childcare is used as a case.

\section{Outcomes}

Table 2 shows an overview of the outcome measures. Those who have not responded by more than a week after the online questionnaire is distributed will receive a popup message to complete each assessment.

Primary outcome

The primary outcome is the onset of MDE during the 32 weeks gestation and 3 months post partum. The onset of

Table 2 Overview of measurements

\begin{tabular}{|c|c|c|c|c|c|c|}
\hline Measurement & Aim & $\begin{array}{l}\text { Baseline } \\
\text { (T1) }\end{array}$ & $\begin{array}{l}32 \text { weeks } \\
\text { gestation } \\
\text { (T2) }\end{array}$ & $\begin{array}{l}34 \text { weeks } \\
\text { gestation } \\
\text { (T3) }\end{array}$ & $\begin{array}{l}\text { 1-week post } \\
\text { partum } \\
\text { (T4) }\end{array}$ & $\begin{array}{l}3 \text { months post } \\
\text { partum } \\
\text { (T5) }\end{array}$ \\
\hline \multicolumn{7}{|l|}{ Primary outcomes } \\
\hline CIDI & $\begin{array}{l}\text { Diagnosis of major } \\
\text { depressive episode }\end{array}$ & $\checkmark$ & $\checkmark$ & & & $\checkmark$ \\
\hline \multicolumn{7}{|l|}{ Secondary outcomes } \\
\hline EPDS & Depressive symptoms & $\checkmark$ & $\checkmark$ & & $\checkmark$ & $\checkmark$ \\
\hline K6 & Psychological distress & $\checkmark$ & $\checkmark$ & & $\checkmark$ & $\checkmark$ \\
\hline EQ-5D-5L & Quality of life & $\checkmark$ & $\checkmark$ & & & $\checkmark$ \\
\hline SSS-8 & Somatic symptoms & $\checkmark$ & $\checkmark$ & & & $\checkmark$ \\
\hline TRS & Resilience & $\checkmark$ & $\checkmark$ & & & $\checkmark$ \\
\hline ISI & Insomnia & $\checkmark$ & $\checkmark$ & & & $\checkmark$ \\
\hline $\mathrm{CH}-\mathrm{RLSq} 13$ & Restless legs syndrome & $\checkmark$ & $\checkmark$ & & & $\checkmark$ \\
\hline $\begin{array}{l}\text { Maternal Anxiety Scale for } \\
4-5 \text { months children }\end{array}$ & Maternal anxiety & & & & & $\checkmark$ \\
\hline Medical costs & Medical service use & $\checkmark$ & $\checkmark$ & & & $\checkmark$ \\
\hline $\begin{array}{l}\text { Implementation and } \\
\text { satisfaction }\end{array}$ & Implementation outcomes & & & $\checkmark$ & & \\
\hline Adverse effects & $\begin{array}{l}\text { Physical and mental } \\
\text { symptoms }\end{array}$ & & & $\checkmark$ & & \\
\hline \multicolumn{7}{|l|}{ Baseline assessments } \\
\hline Demographics & & $\checkmark$ & & & & \\
\hline VAWS & & $\checkmark$ & & & & \\
\hline
\end{tabular}

CH-RLSq13, Cambridge-Hopkins Questionnaire Short Form; CIDI, WHO Composite International Diagnostic Interview 3.0; ED-5D-5L, EuroQol-5 dimension-5 level; EPDS, Edinburgh Postnatal Depression Scale; ISI, Insomnia Severity Index; K6, Kessler's Psychological Distress Scale; SSS-8, Somatic Symptom Scale-8; TRS, Tachikawa Resilience Scale; VAWS, Violence Against Women Screen. 
MDE during the follow-up will be assessed using the webbased self-administered version of the Japanese WHOCIDI 3.0 depression section, according to Diagnosticand Statistical Manual of Mental Disorders Fourth Edition (Text Revision).

criteria. The web version has been shown to have a good concordance with the clinical diagnosis of MDE and to be reliable in a 1-year test-retest survey. An incident case with MDE will be identified if a respondent reports an episode of MDE at either 32 weeks gestation or 3 months post partum. An onset month for an episode of MDE also will be requested. In addition, two other definitions are applied to identify subthreshold depressive episodes: one requires a shorter duration of symptoms (ie, 7 days or more, rather than 2 weeks or more); the other one requires having a fewer symptoms (ie, having three symptoms or more, instead of five symptoms or more).

\section{Secondary outcomes}

\section{Edinburgh Postnatal Depression Scale (EPDS)}

Depressive symptoms will be measured by the Japanese version of EPDS. ${ }^{31}{ }^{32}$ EPDS is used most often for screening perinatal depression because it focuses on cognitive symptoms of depression and excludes somatic items that can generate false positives during pregnancy and post partum. It consists of 10 items, with $0-3$ points scored per item for a potential scale score of $0-30$. The higher scores indicate more severe depressive symptoms. EPDS will be conducted at baseline, 32 weeks gestation, 1-week post partum and 3 months post partum.

\section{Kessler's Psychological Distress Scale}

Psychological distress is measured by the Japanese version of $\mathrm{K} 6 .{ }^{33}{ }^{34} \mathrm{~K} 6$ consists of six items assessing the frequency with which respondents have experienced symptoms of psychological distress during the past 30 days. The response options range from 0 (none of the time) to 4 (all of the time), and the total score ranges from 0 to 24. The higher scores indicate more severe psychological distress. K6 will be conducted at baseline, 32 weeks gestation, 1-week post partum and 3 months post partum.

\section{EuroQol-5 dimension-5 level (EQ-5D-5L)}

General health status will be measured by the Japanese version of EQ-5D-5L. ${ }^{35-37}$ It is a five-dimensional utility instrument consisting of domains about morbidity, selfcare, usual activities, pain or discomfort, and anxiety or depression. Each domain is divided into five levels of severity (none, slight, moderate, severe, extreme problems or unable to). All responses are converted into a single index score of general health status. EQ-5D-5L will be conducted at baseline, 32 weeks gestation and 3 months post partum.

\section{Somatic Symptom Scale-8 (SSS-8)}

Somatic symptoms will be measured by Japanese version of SSS- $8 .{ }^{38}{ }^{39}$ SSS- 8 consists of eight items that assess the following symptoms: stomach or bowel problems; back pain; pain in the arms, legs, or joints; headaches; chest pain or shortness of breath; dizziness; feeling tired or having low energy; and trouble sleeping. These items comprise the four symptom domains of gastrointestinal, pain, cardiopulmonary and fatigue. Respondents rate how much each symptom has bothered them during the previous 7 days and score each item from 0 to 4 : not at all (0), a little bit (1), somewhat (2), quite a bit (3) and very much (4). The total score ranges from 0 to 32 . A higher score reflects a more severe somatic symptom burden. SSS-8 will be conducted at baseline, 32 weeks gestation and 3 months post partum.

\section{Tachikawa Resilience Scale (TRS)}

Resilience will be measured by TRS. ${ }^{40}$ TRS consists of 10 items. All items are scored on a 7-point scale from 1 (strongly disagree) to 7 (strongly agree), with a total score ranging from 10 to 70 . Higher scores reflect higher resilience. TRS will be conducted at baseline, 32 weeks gestation and 3 months post partum.

\section{Insomnia Severity Index (ISI)}

Insomnia will be measured by the Japanese version of ISI. ${ }^{41}{ }^{42}$ ISI consists of seven items assessing the severity of sleep-onset and sleep maintenance difficulties (both nocturnal and early morning awakenings), satisfaction with the current sleep pattern, interference with daily functioning, awareness of impairment attributed to the sleep problem and degree of distress or concern caused by the sleep problem. Each item is rated on a $0-4$ scale and the total score ranges from 0 to 28 . A higher score suggests more severe insomnia. ISI will be conducted at baseline, 32 weeks gestation and 3 months post partum.

\section{Cambridge-Hopkins Questionnaire Short Form (CH-RLSq13)}

Restless legs syndrome (RLS) will be measured by the Japanese version of CH-RLSq13. ${ }^{43}$ CH-RLSq13 consists of 13 items. Diagnosis of RLS is performed using 10 items consisting of questions about discomfort and stiffness of the lower limbs. In addition, two items ask about the degree of pain and the frequency of occurrence and one item asks about the age of onset (relevant to pregnancy in women). CH-RLSq13 will be conducted at baseline, 32 weeks gestation and 3 months post partum.

\section{Maternal Anxiety Scale for 4-5-month-old children}

Maternal anxiety will be measured by the Maternal Anxiety Scale for $4-5$ month-old children. ${ }^{45}$ This scale consists of 34 items. Eleven items assess childcare anxiety, six items assess husbands' or partners' support, five items assess childcare satisfaction, four items assess ease of raising children, five items assess a lack of confidence and three items assess presence or absence of advisors. All items are scored on a 4-point scale from 1 (strongly disagree) to 4 (strongly agree). A higher score of each subscale reflects a higher presence of each factor. This scale will be conducted at 3 months post partum. 


\section{Medical economic costs}

For cost-effectiveness analysis, the presence or absence, frequency and duration of medical service use, and the use of drugs over the prior 3 months will be asked at 32 weeks gestation and 3 months post partum.

\section{Process evaluation}

\section{Implementation outcomes}

Time spent logged in to each module will be measured. Also, we will evaluate implementation outcomes by selfreport via survey. Proctor et al suggested eight conceptually distinct implementation outcomes: acceptability, adoption, appropriateness, feasibility, fidelity, implementation cost, penetration and sustainability. ${ }^{46}$ However, a systematic review examined implementation outcomes for mental health and behavioural health using evidencebased rating criteria and concluded that the majority of instrumental outcomes were underdeveloped. ${ }^{47}$ Recently, Weiner et al developed measures of acceptability, appropriateness and feasibility because their outcomes are often used as leading indicators and are conceptually distinct. ${ }^{48}$ Three of the authors (DN, EO and NS) reviewed the previous literature and selected the possible outcomes for each dimension of acceptability, appropriateness and feasibility. These implementation outcomes and satisfaction with the intervention programme will be asked about at 34 weeks gestation.

\section{Adverse effects}

Three of the authors (DN, EO and NS) reviewed the previous literature based on a systematic review, and selected the possible adverse effects of the programme, such as physical symptoms (eg, tired eyes, stiff shoulders), mental symptom (eg, insomnia), dangerous experiences (eg, collide with people while walking and looking at the smartphone), too much use of the smartphone and excessive pressure to learn this programme regularly. These potential adverse effects will be asked about at 34 weeks gestation.

\section{Data collection}

All data will be collected by the internet. If the entered data are incomplete, participants will not be able to proceed the assessment.

\section{Sample size calculation}

Required sample size was calculated for the primary outcome. New onset of MDE during the observation period in Japan and the effect size in HR are estimated to be $5 \%$ and $0.65,{ }^{49}$ based on previous studies. Thirty per cent of participants are expected to drop out of the follow-up assessment. Given an $\alpha$ level of 0.05 (two-tailed) and a $\beta$ level of 0.20 , power Cox was performed with STATA V.14.0 and the appropriate sample size was calculated to be 4812. Since a large number of participants using the app will be recruited on 1 day, it is considered difficult to stop recruiting immediately when the sample size is reached. Thus, the sample size was set at 5000 .

\section{Randomisation}

Participants who meet the inclusion criteria will be randomly allocated to the intervention group or control group. Participants will be stratified into two strata according to the score of K6 (four or less, or five or more) on the baseline survey. MTI will send baseline data to researchers. In addition to the analysis of the whole sample (to examine the universal intervention effect), we will also analyse data by prespecified subgroups (to examine the selective intervention effect). Using a computer-generated random allocation sequence, an independent biostatistician created a stratified permutedblock random table. The block size of this RCT will be fixed at 4 . The stratified permuted-block random table will be password protected and blinded to the researcher. Only the research assistant will be able to access it during the work of random allocation. MTI will make the study participants allocated to the intervention group available to view the iCBT programme on the app, based on the allocation provided by the research assistant.

\section{Statistical methods}

Main analysis

As described in detail previously, ${ }^{50} 51$ a survival analysis will be conducted to test for the effectiveness of the intervention on the time to the onset of MDE while controlling for censoring effects due to the differential length of follow-up or the completion of follow-up without an onset of MDE. Length of follow-up for each participant will be represented by either the number of months between the baseline and the onset of MDE or the end of the follow-up period (3 months post partum, or 32 weeks gestation if a participant dropped out at the 3-month postpartum follow-up), whichever comes first. The cumulative incidence of MDE at the 32 weeks gestation and 3-month postpartum follow-up, as well as event-free survivals at every follow-up month, will be estimated using the Kaplan-Meier method; the statistical significance will be tested of the difference between the cumulative proportions of having MDE at 32 weeks gestation and 3-month postpartum follow-ups in the intervention and control groups. A log-rank test will be conducted to test the difference in survival probabilities between the intervention and the control groups. A single covariate Cox discrete time hazard model will also be used to test the difference and estimate the HR, with 95\% CIs, for having MDE in the intervention group compared with the control group. The intervention effect will also be estimated, adjusting for dependent censoring and using the inverse probability of the censoring weighted method for conducting a sensitivity analysis. ${ }^{52}$ The number needed to treat to achieve prevention of one case of the onset of MDE will be calculated at 32 weeks gestation and 3-month postpartum follow-ups. A similar Cox discrete time hazard model also will be conducted using the two types of subthreshold diagnoses of depressive episodes to investigate the effects of iCBT on preventing depressive episodes, including 
subclinical cases. An intention-to-treat (ITT) analysis will be conducted. Multiple imputation will be performed.

\section{Secondary analyses}

For secondary outcomes (ie, EPDS, K6, SSS-8, TRS), mixed models for repeated measures analyses will be conducted using a group (intervention or control) $x$ time (baseline, 32 weeks gestation, 1-week postpartum or 3-month postpartum follow-up) interaction as an indicator of the intervention effect. This allows for missing data to be taken into account within the statistical model. The level of statistical significance for all analyses in this study will be set at 0.05 (two-tailed), and $95 \%$ CIs will be calculated. The effect size will be estimated in two ways. First, we will estimate a regression coefficient for a group (the intervention group vs the control group) $\times$ time (baseline and follow-ups) interaction using the mixed procedure, which will be converted to an effect size by dividing by a pooled SD at baseline and at follow-ups. Second, we will calculate Cohen's d among completers at baseline for each follow-up. ITT will be conducted as well. All statistical analyses will be conducted using SPSS Statistics V.21.0 (IBM Corp.).

\section{Subgroup analysis}

The effectiveness of the programmes may differ according to the initial severity of psychological distress. Therefore, we will analyse the results according to the prespecified subgroups (ie, participants who scored 4 or less $/ 5$ or more in $\mathrm{K} 6$ at the baseline survey).

\section{Cost-effectiveness analysis}

Quality-adjusted life-years will be calculated as the effectiveness, using the EQ-5D-5L, as the area under the curve defined by the unitality values at baseline and follow-ups. ${ }^{53}$ As for the costs, the cost of medical service use will be calculated based on the Survey of Medical Care Activities in Public Health Insurance, ${ }^{54}$ which shows the treatment expenses covered by public health insurance in each diagnostic category. No intervention costs such as salary for therapists will be calculated because the programme is fully automated. Mean differences for the calculated effectiveness and costs will be compared between the intervention and control group.

\section{Data monitoring and auditing}

Because the iCBT programme is not regarded as an invasive intervention, it is not necessary to set up a data monitoring board or to complete auditing in this trial.

\section{Patient and public involvement}

We used the function of the app Luna Luna Baby that allows users (pregnant women) to talk to each other about their problems. We extracted essential topics that pregnant women are concerned about based on 6393 text data, and developed programmes for those topics. Even MTI cannot identify the person who posted a text, the text data are anonymised data that cannot be linked. The procedure was approved by the ethic committee of the
University of Tokyo. The topics were extracted by using a machine learning approach, based on the latent Dirichlet allocation (LDA). ${ }^{55}$ In this approach, morphological analysis was conducted for the text data from the pregnant women, and nouns and adjectives about what they suffer were collected. The LDA model was implemented by Scikit-learn V.0.21. $3^{56}$ in Python. As a result, five topics were extracted: relationship with partners, concern about weight gain, concern about pregnancy check-up, physical symptoms such as pain and dysfunction due to morning sickness. Since pregnancy check-up is considered to be a matter of obstetrics, each module of the iCBT programme has been developed to deal with the remaining four topics. We will not access this chat room during the RCT.

In addition, three women who had experiences of pregnancy and childbirth (two researchers and a research partner with lived experience) were invited to make comments on the intervention programmes based on their experiences and preferences. All of them experienced maternity blues or perinatal depression, though they did not visit psychiatrists. Two researchers above (EO and NS) also were involved in designing this protocol.

\section{Ethics and dissemination}

\section{Ethical and safety considerations}

Informed consent for the app will be obtained from all participants included in this study after full explanation of the study. Candidates will be informed that their participation is voluntary, and that even after voluntarily participating, they can withdraw from the study at any time and their withdrawal will cause no disadvantage to them. We expect no adverse health effects from this intervention, except possible deterioration in depressive symptoms. We will send messages to those who meet the criteria for MDE in the past month or for lifetime bipolar disorders at baseline to encourage them to see a psychiatrist. The principal investigators will communicate important protocol modification with the institutional review board.

\section{Data confidentiality}

The collected data will be stored as linkable anonymising data. The principal investigator will have access to the final dataset after the trial and take responsibility for the integrity of the data and the accuracy of analysis.

\section{Dissemination of research findings}

The findings of this study will be disseminated through publications in peer-reviewed international journals. Presentations of the findings will also be offered at relevant research conferences, and local academic symposia and seminars. If important findings are obtained from this study, we will make a press release and provide a plain language summary for users of Luna Luna Baby. The principal investigator will be listed as corresponding authors, and the authorship eligibility will be conformed to the International Committee of Medical Journal Editors. If the intervention programmes are found to be 
significantly positively effective, the programmes can be made available for all users of the app in the future.

\section{DISCUSSION}

The greatest strength of this study is to prove the effectiveness of the fully automated smartphone-based CBT programme on preventing both antenatal and postpartum depression. The relatively short, newly developed programmes for pregnant women would be practical and low dropout is expected. Furthermore, this study will assess diagnosis of MDE using CIDI. Many previous studies using iCBT have suggested its preventive effect on depression; however, no previous studies assessed the diagnosis of perinatal depression using the structural interview. This RCT with large sample size can lead to a definitive result.

This study also will evaluate implementation outcomes of the programme. Previous studies using iCBT have assessed the satisfaction with their programme; however, few studies evaluated implementation outcomes including acceptability, adoption, appropriateness, feasibility, fidelity, implementation cost, penetration and sustainability. ${ }^{46}$ This study will contribute to the dissemination and implementation of iCBT in the future.

Another strength of this study is to add evidence of maternity blues. Maternity blues is highly prevalent, and it can present a range of symptoms such as intense short-lasting dysphoric mood, irritability, anxiety, sleep disturbance and poor concentration within the first week following childbirth. ${ }^{57}$ Maternity blues could lead to postpartum depression, ${ }^{58}$ but to our knowledge, no previous studies have shown a preventive strategy to deal with maternity blues. This RCT will clarify whether iCBT during pregnancy can prevent maternity blues.

This study has several limitations. First, all outcomes will be measured by self-report, which could be affected by the perceptions of the participants. Second, users of the app are not regarded as representative of all pregnant women, though approximately one in four pregnant women in Japan is thought to use the app. Therefore, the findings of this study cannot necessarily be generalisable. Third, follow-up period is not long enough, because a sizeable proportion of postpartum depression have onset after 3 months post partum.

Contributors DN conceived and designed the study. DN, KI, EO, NS and YS contributed creating programmes. NY and NK contributed to the development of study design. KW calculated sample size. KW and YM developed analysis plan. DN wrote the first draft of the manuscript, and all other authors revised the manuscript critically. All authors approved the final version of the manuscript.

Funding This work was supported by Japan Society for the Promotion of Science under a Grant-in-Aid for Scientific Research (A) (19H01073 to DN).

Disclaimer The funder had no role in study design, data collection and analysis, decision to publish or preparation of the manuscript.

Competing interests MTI has been involved in this study as mentioned in the manuscript. NK reports grants from Infocom Corp, Fujitsu, Fujitsu Software Technologies and TAK; personal fees from Occupational Health Foundation, Japan Dental Association, Sekisui Chemicals, Junpukai Health Care Center, Osaka Chamber of Commerce and Industry, outside the submitted work.
Patient and public involvement Patients and/or the public were involved in the design, or conduct, or reporting, or dissemination plans of this research. Refer to the Methods section for further details.

\section{Patient consent for publication Obtained.}

Provenance and peer review Not commissioned; externally peer reviewed.

Open access This is an open access article distributed in accordance with the Creative Commons Attribution Non Commercial (CC BY-NC 4.0) license, which permits others to distribute, remix, adapt, build upon this work non-commercially, and license their derivative works on different terms, provided the original work is properly cited, appropriate credit is given, any changes made indicated, and the use is non-commercial. See: http://creativecommons.org/licenses/by-nc/4.0/.

\section{ORCID iDs}

Daisuke Nishi http://orcid.org/0000-0001-9349-3294

Naonori Yasuma http://orcid.org/0000-0002-1216-7639

Norito Kawakami http://orcid.org/0000-0003-1080-2720

\section{REFERENCES}

1 Wisner KL, Miller ES, Tandon D. Attention to prevention-can we stop perinatal depression before it starts? JAMA Psychiatry 2019;76:355-6.

2 Bennett HA, Einarson A, Taddio A, et al. Prevalence of depression during pregnancy: systematic review. Obstet Gynecol 2004;103:698-709.

3 Hahn-Holbrook J, Cornwell-Hinrichs T, Anaya I. Economic and health predictors of national postpartum depression prevalence: a systematic review, meta-analysis, and meta-regression of 291 studies from 56 countries. Front Psychiatry 2017;8:248.

4 Takehara K, Suto M, Kakee N, et al. Prenatal and early postnatal depression and child maltreatment among Japanese fathers. Child Abuse Negl 2017;70:231-9.

5 Davis EP, Glynn LM, Schetter CD, et al. Prenatal exposure to maternal depression and cortisol influences infant temperament. J Am Acad Child Adolesc Psychiatry 2007;46:737-46.

6 Leech SL, Larkby CA, Day R, et al. Predictors and correlates of high levels of depression and anxiety symptoms among children at age 10. J Am Acad Child Adolesc Psychiatry 2006;45:223-30.

7 Field T, Diego M, Hernandez-Reif M. Prenatal depression effects on the fetus and newborn: a review. Infant Behav Dev 2006;29:445-55.

8 Diego MA, Field T, Hernandez-Reif M, et al. Prenatal depression restricts fetal growth. Early Hum Dev 2009;85:65-70.

9 Stein A, Pearson RM, Goodman SH, et al. Effects of perinatal mental disorders on the fetus and child. Lancet 2014;384:1800-19.

10 Underwood L, Waldie KE, Peterson E, et al. Paternal depression symptoms during pregnancy and after childbirth among participants in the growing up in New Zealand study. JAMA Psychiatry 2017;74:360-9.

11 Lancaster CA, Gold KJ, Flynn HA, et al. Risk factors for depressive symptoms during pregnancy: a systematic review. Am J Obstet Gynecol 2010;202:5-14.

12 US Preventive Services Task Force, Curry SJ, Krist AH, et al. Interventions to prevent perinatal depression: US preventive services Task force recommendation statement. JAMA 2019;321:580-7.

13 Sockol LE. A systematic review of the efficacy of cognitive behavioral therapy for treating and preventing perinatal depression. J Affect Disord 2015;177:7-21.

14 Sockol LE. A systematic review and meta-analysis of interpersonal psychotherapy for perinatal women. J Affect Disord 2018;232:316-28.

15 Sockol LE, Epperson CN, Barber JP. Preventing postpartum depression: a meta-analytic review. Clin Psychol Rev 2013;33:1205-17.

16 Fairburn CG, Patel V. The impact of digital technology on psychological treatments and their dissemination. Behav Res Ther 2017;88:19-25.

17 Loughnan SA, Joubert AE, Grierson A, et al. Internet-Delivered psychological interventions for clinical anxiety and depression in perinatal women: a systematic review and meta-analysis. Arch Womens Ment Health 2019;22:737-50.

18 Barrera AZ, Wickham RE, Muñoz RF. Online prevention of postpartum depression for Spanish- and English-speaking pregnant women: a pilot randomized controlled trial. Internet Interv 2015;2:257-65. 
19 Haga SM, Drozd F, Lisøy C, et al. Mamma Mia - A randomized controlled trial of an internet-based intervention for perinatal depression. Psychol Med 2019;49:1850-8.

20 Imamura K, Kawakami N, Furukawa TA, et al. Does Internet-based cognitive behavioral therapy (iCBT) prevent major depressive episode for workers? A 12-month follow-up of a randomized controlled trial. Psychol Med 2015;45:1907-17.

21 Henshaw C, Foreman D, Cox J. Postnatal blues: a risk factor for postnatal depression. J Psychosom Obstet Gynaecol 2004;25:267-72.

22 Chan A-W, Tetzlaff JM, Gøtzsche PC, et al. Spirit 2013 explanation and elaboration: guidance for protocols of clinical trials. BMJ 2013;346:e7586.

23 Kessler RC, Ustün TB. The world mental health (WMH) survey initiative version of the world Health organization (who) composite international diagnostic interview (CIDI). Int J Methods Psychiatr Res 2004;13:93-121.

24 Padesky CA, Greenberger D. Clinician's guide to mind over mood. New York: Guilford Press, 1995.

25 Cassar J, Ross J, Dahne J, et al. Therapist tips for the brief behavioural activation therapy for depression - revised (BATD-R) treatment manual practical wisdom and clinical nuance. Clin Psychol 2016;20:46-53.

26 Hayes SC, Stroshl K, Wilson KG. Acceptance and commitment therapy: an experiential approach to behavior change. New York: Guilford Press, 1999.

27 Gilbert P. The compassionate mind: a new approach to life's challenges. Constable \& Robinson: Oakland, 2009.

28 Zessin U, Dickhäuser O, Garbade S. The relationship between selfcompassion and well-being: a meta-analysis. Appl Psychol Health Well Being 2015;7:340-64.

29 Kabat-Zinn J, Go WY. There you are: mindfulness meditation in everyday life. New York: Hyperion Books, 1994.

30 D'Zurilla TJ, Goldfried MR. Problem solving and behavior modification. J Abnorm Psychol 1971;78:107-26.

31 Cox JL, Holden JM, Sagovsky R. Detection of postnatal depression. development of the 10-item Edinburgh postnatal depression scale. Br J Psychiatry 1987;150:782-6.

32 Okano T, Murata M, Masuji F, et al. Validation and reliability of Japanese version of the EPDS. Arch Psychiatr Diag Clin Evaluat 1996;7:525-33.

33 Kessler RC, Andrews G, Colpe LJ, et al. Short screening scales to monitor population prevalences and trends in non-specific psychological distress. Psychol Med 2002;32:959-76.

34 Furukawa TA, Kawakami N, Saitoh M, et al. The performance of the Japanese version of the K6 and K10 in the world mental health survey Japan. Int J Methods Psychiatr Res 2008;17:152-8.

35 Herdman M, Gudex C, Lloyd A, et al. Development and preliminary testing of the new five-level version of EQ-5D (EQ-5D-5L). Qual Life Res 2011;20:1727-36.

36 van Hout B, Janssen MF, Feng Y-S, et al. Interim scoring for the EQ$5 \mathrm{D}-5 \mathrm{~L}$ : mapping the EQ-5D-5L to EQ-5D-3L value sets. Value Health 2012;15:708-15.

37 Ikeda S, Shiroiwa T, Igarashi A, et al. Developing a Japanese version of the EQ-5D-5L value set. J Natl Inst Public Health 2015;64:47-55.

38 Gierk B, Kohlmann S, Kroenke K, et al. The somatic symptom scale-8 (SSS-8): a brief measure of somatic symptom burden. JAMA Intern Med 2014;174:399-407.

39 Matsudaira K, Oka H, Kawaguchi M, et al. Development of a Japanese version of the somatic symptom Scale-8: psychometric validity and internal consistency. Gen Hosp Psychiatry 2017;45:7-11.

40 Nishi D, Uehara R, Yoshikawa E, et al. Culturally sensitive and universal measure of resilience for Japanese populations: Tachikawa resilience scale in comparison with resilience scale 14-item version. Psychiatry Clin Neurosci 2013;67:174-81.

41 Bastien $\mathrm{CH}$, Vallières $\mathrm{A}$, Morin $\mathrm{CM}$. Validation of the insomnia severity index as an outcome measure for insomnia research. Sleep Med 2001;2:297-307.

42 Munezawa T, Morin CM, Inoue Y. Development of the Japanese version of the insomnia severity index (ISI-J) Seishinkachiryogaku. 2009: 24, 219-25.

43 Allen RP, Burchell BJ, MacDonald B, et al. Validation of the selfcompleted Cambridge-Hopkins questionnaire (CH-RLSq) for ascertainment of restless legs syndrome (RLS) in a population survey. Sleep Med 2009;10:1097-100.

44 Kondo H, Okada M, Eto H. Development of Cambridge-Hopkins questionnaire 13 Japanese version short form (CH-RLSq13) version 2.1 in the assessment of restless legs syndrome (in Japanese), 2016: 577-84.

45 Yoshida H, Yamanaka T, Khono G. The study for the development of maternal anxiety scales-1: models for mothers rearing $4 \cdot 5$ monthold and 10. 11 month-old infants, 2013: 680-9.

46 Proctor E, Silmere H, Raghavan R, et al. Outcomes for implementation research: conceptual distinctions, measurement challenges, and research agenda. Adm Policy Ment Health 2011;38:65-76.

47 Lewis CC, Fischer S, Weiner BJ, et al. Outcomes for implementation science: an enhanced systematic review of instruments using evidence-based rating criteria. Implement Sci 2015;10:155.

48 Weiner BJ, Lewis CC, Stanick C, et al. Psychometric assessment of three newly developed implementation outcome measures. Implement Sci 2017;12:108.

49 Cuijpers P, van Straten A, Smit F, et al. Preventing the onset of depressive disorders: a meta-analytic review of psychological interventions. Am J Psychiatry 2008;165:1272-80.

50 Imamura K, Kawakami N, Furukawa TA, et al. Effects of an Internetbased cognitive behavioural therapy intervention on preventing major depressive episodes among workers: a protocol for a randomised controlled trial. BMJ Open 2015;5:e007590.

51 Imamura K, Tran TTT, Nguyen HT, et al. Effects of two types of smartphone-based stress management programmes on depressive and anxiety symptoms among hospital nurses in Vietnam: a protocol for three-arm randomised controlled trial. BMJ Open 2019;9:e025138.

52 Robins JM, Finkelstein DM. Correcting for noncompliance and dependent censoring in an AIDS clinical trial with inverse probability of censoring weighted (IPCW) log-rank tests. Biometrics 2000;56:779-88.

53 Richards DA, Ekers D, McMillan D, et al. Cost and outcome of behavioural activation versus cognitive behavioural therapy for depression (cobra): a randomised, controlled, non-inferiority trial Lancet 2016;388:871-80.

54 Ministry of Health WaL. Survey of medical care activities in public health insurance in 2016 (in Japanese), 2016. Available: https://www. mhlw.go.jp/toukei/saikin/hw/sinryo/tyosa16/

55 Blei DM, AY N, Jordan MI, et al. Latent Dirichlet allocation. J Mach Learn Res 2003:993-1022.

56 Scikit-learn. Latent Dirichlet allocation. Available: https://scikit-learn. org/stable/modules/generated/sklearn.decomposition.LatentDirich letAllocation.html

57 Kennerley H, Gath D. Maternity blues. I. detection and measurement by questionnaire. Br J Psychiatry 1989;155:356-62.

58 Hannah P, Adams D, Lee A, et al. Links between early postpartum mood and post-natal depression. Br J Psychiatry 1992;160:777-80. 